\title{
Ist eine systemische Antibiotikatherapie nach Abszeßtonsillektomie erforderlich?
}

\author{
St. Knipping, St. Deumer, M. Bloching
}

Martin-Luther-Universität Halle-Wittenberg Universitätsklinik und Poliklinik

für HNO-Heilkunde, Kopf- und Halschirurgie

(Kommissarischer Leiter: PD Dr. med. M. Bloching)

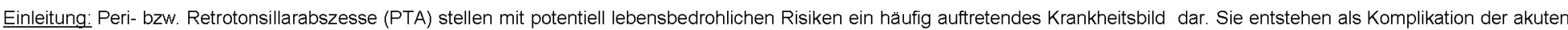

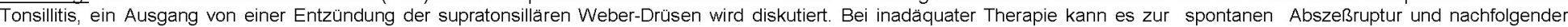

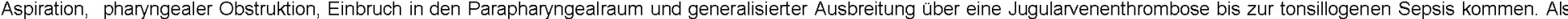

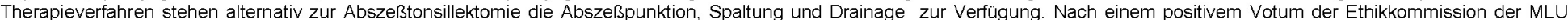
Halle-Wittenberg sollte in dieser Studie die Notwendigkeit einer postoperativen Antibiotikagabe nach Abszeßtonsillektomie überprüft werden.

Material und Methode: Im Zeitraum von Januar 1996 - Dezember 2003 wurden 283 Patienten mit einem Peritonsillarabszeß (PTA) behandelt.

Geschlechtsverteilung: 98 weibliche (34,6\%) / 185 männliche (65,4\%) Patienten

Altersverteilung: Patienten vom 2. bis 72 . Lebensjahr (siehe Diagramm1)

Ausschlußkriterien: Patienten <18. Lebensjahr, kardiale Erkrankungen, Immuninkompetenz, rheumatische Erkrankungen, Nephropathien, Autoimmunerkrankungen

Symptome: bei 283 Patienten Peritonsillarschwellung, bei 247 Patienten Uvulaodem, bei 235 Patienten Kieferklemme, bei 107 Patienten Fieber

Art der Behandlung: stationäre Betreuung, Abszeßtonsillektomie am Aufnahmetag,

postoperativ Benzypenizillin für 10 Tage (Gruppe1), postop. keine Antibiotikagabe (Gruppe2) Analgesie mit Parazetamol

Anamnese: vor dem PTA traten auf: bei 76 Patienten chronische Tonsillitis, bei 8 Patienten ein vorheriger PTA, bei 1 Patient eine chronische Tonsillitis mit PTA, bei 198 Patienten trat ein Primär-PTA auf
Diagramm 1

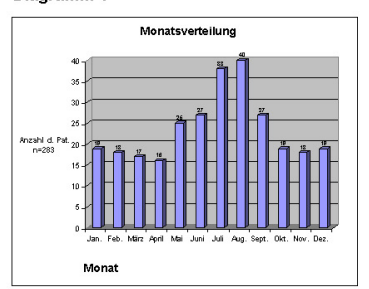

Diagramm 2

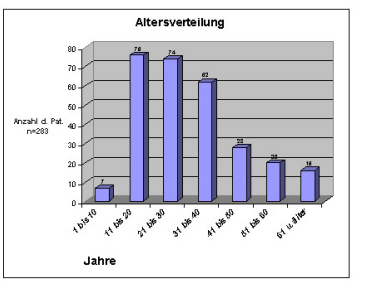

Schmerzen

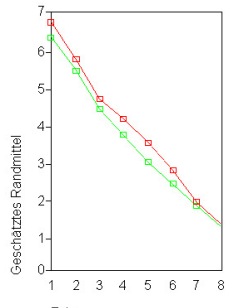

Zoit
Schluckbeschwerden

Wohlbefinden

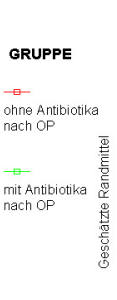

Diagram

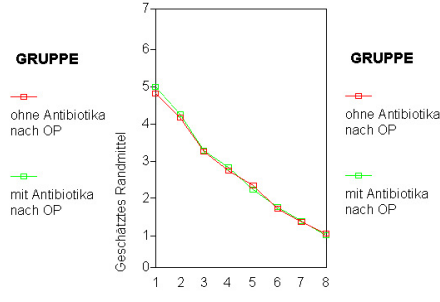

Zeit

ie entnommenen Blutkulturen zeigten in beiden Gruppen kein Keimwachstum

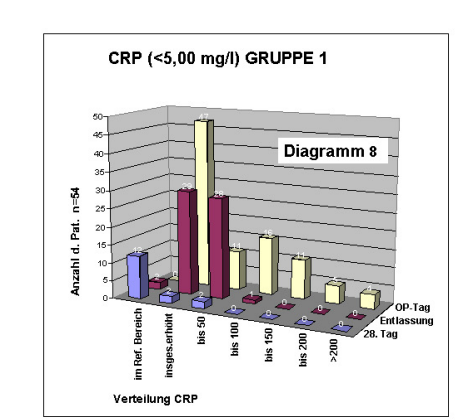

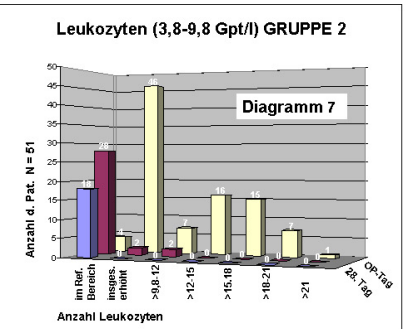

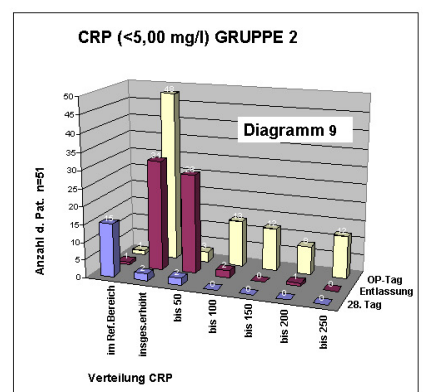

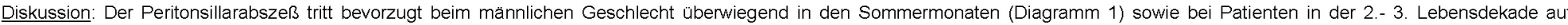

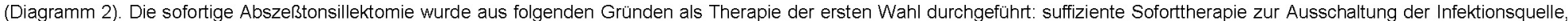

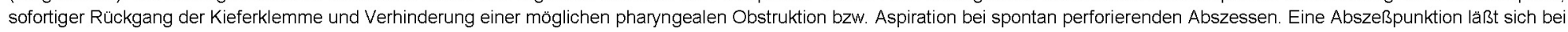

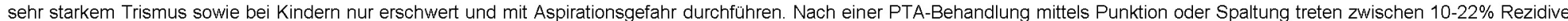

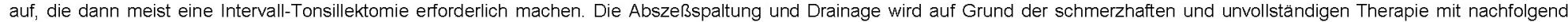
erforderlicher Tonsillektomie in unserer Klinik nicht durchgeführt.

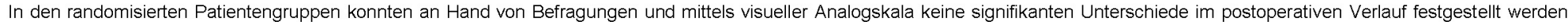

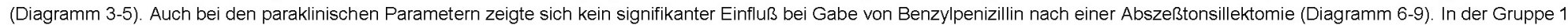

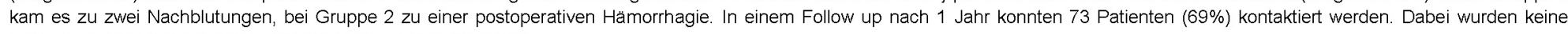
Besonderheiten (Schmerzen, chronische Pharyngitis) registriert.

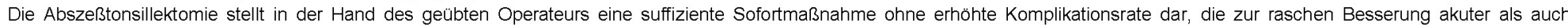

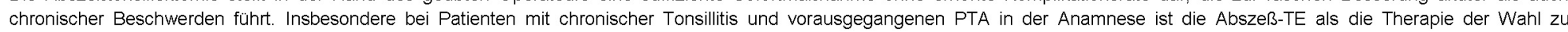

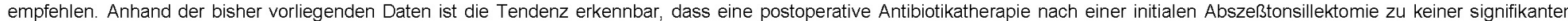

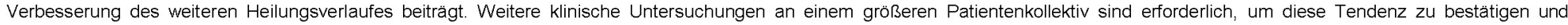
Therapieempfehlungen daraus abzuleiten.

Literatur:

1) Chowdhury, C.R., Bricknell, B.M.: The management of quinsy-a prospective study. The journal of Laryngology and Otology, vol. 106 (1992),986-988 2) Harley, E.H.: Quinsy Tonsillectomy as the Treatment of Choice for Peritonsillar Abscess. Ear, Nose and Throat Journal 67 (1988),84-87

3) Herzon, F.S.: Peritonsillar Abscess: Incidence, Current Management Practices and a Proposal for Treatment Guidelines. Laryngoscope 105 (1995), 1-17

4) Johnson, R.F., Stewart, M.G., Wright, C.C.: An evidence-based review of the treatment of peritonsillar abscess. Otolaryngol Head Neck Surgery 128 (2003), $332-343$

5) Knipping, St., Passmann, M., Schrom, T., Berghaus, A.: Abscess tonsillctomy for acute peritonsillar abscess. Rev Laryngol Otol Rhinol 123 (2002), 13-16

6) Lockhart, R, Parker, G.S.: Role of quinsy tonsillectomy in the management of PTA. Ann Otol Rhinol Laryngol 100 (1991),569-571

7) Passy, V.: Pathogenesis of Peritonsillar Abscess. Laryngoscope 104 (1994), 185-190

8) Windfuhr, J.P., Remmert, S.: Peritonsillarabszess: Trends und Komplikationen bei der Behandlung, insbesondere bei Kindern. HNO 53 (2005), 46-57 